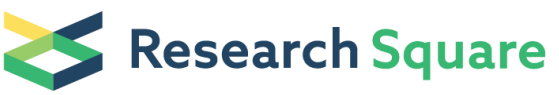 \\ Preprints are preliminary reports that have not undergone peer review. \\ They should not be considered conclusive, used to inform clinical practice, or referenced by the media as validated information.
}

\section{Nutritional, Lipid Metabolic and Body Composition Features of Children with Spinal Muscular Atrophy}

\section{Yijie Feng}

Zhejiang University School of Medicine Children's Hospital

\section{Mei Yao}

Zhejiang University School of Medicine Children's Hospital

Yu Xia

Zhejiang University School of Medicine Children's Hospital

Jia Wei

Zhejiang University School of Medicine Children's Hospital

Yi Hong

Zhejiang University School of Medicine Children's Hospital

\section{Qi Long}

Zhejiang University School of Medicine Children's Hospital

\section{Fei Chen}

Zhejiang University School of Medicine Children's Hospital Jianing Jin

Zhejiang University School of Medicine Children's Hospital

Dongming Zhou

Zhejiang University School of Medicine Children's Hospital

Jingjing $\mathrm{Hu}$

Zhejiang University School of Public Health

Yiqin Cui

Zhejiang University School of Medicine Children's Hospital

Xiao Chen

Zhejiang University School of Public Health

Changzheng Yuan

Zhejiang University School of Medicine Children's Hospital

Shanshan Mao ( $6307003 @ z j u . e d u . c n$ )

Zhejiang University School of Medicine Children's Hospital https://orcid.org/0000-0001-6736-1407

\section{Research Article}

Keywords: Spinal muscular atrophy, nutritional status, lipid metabolism, body composition, DXA 
Posted Date: November 19th, 2021

DOI: https://doi.org/10.21203/rs.3.rs-1071489/v1

License: (c) (i) This work is licensed under a Creative Commons Attribution 4.0 International License. Read Full License 


\section{Abstract \\ Background}

Spinal Muscular Atrophy (SMA) is a progressive neuromuscular disease affecting multiple organs and systems, including nutritional and metabolic status. There is still a lack of relevant large sample size research. We aimed to investigate the nutritional status, serum lipid profile, and body composition characteristics in children with SMA.

\section{Methods}

A hospital-based, case-control study was conducted on 91 children with SMA (17.6\% of type I, $48.4 \%$ of type II, $34.1 \%$ of type III) and 91 age- and gender-matched healthy children in a single hospitalized center in China. Anthropometric information, serum lipids parameters and body composition (in subgroup of patients older than 3 years) were collected. According to the anthropometric Z scores, nutritional status was categorized into normal and malnutrition (including under- and over-nutrition) groups.

\section{Results}

The prevalence of malnutrition and dyslipidemia in children with SMA was higher than that of controls ( $49.45 \%$ vs. $23.08 \%$ and $56.04 \%$ vs. $34.07 \%$, respectively and $p<0.01)$. Particularly, the prevalence of lower-than-normal levels of high density lipoprotein (HDL) and apolipoprotein A1 (Apo A1) were significantly higher than that in healthy controls in children with SMA ( $26.37 \%$ vs. $7.69 \%$ and $41.76 \%$ vs. $6.59 \%$, respectively and $p<0.01)$. In terms of the body composition characteristics in children with SMA II \& III, on average, the total body fat mass percentage (FM\%) was $43.38 \%(36.99 \%, 48.20 \%)$, the fat mass index (FMI) was $7.30(5.07,8.72)$, the lean body mass index (LBMI) was $7.70(6.96,9.02)$ and the bone mineral density-z score (BMD-z) was $-1.40(-2.80,-0.60)$, generally different from the reference profiles in published literature.

\section{Conclusion}

Children with SMA in China have obvious nutritional imbalances, abnormal levels of serum lipid and unbalanced body composition. Further research is warranted to explore the potential mechanisms and health management strategies on nutritional and metabolic disorders of SMA.

\section{Background}

Spinal muscular atrophy (SMA) is an autosomal recessive genetic neuromuscular disorder that affects the anterior horn cells of the spinal cord and is characterized by spinal motor neuron degeneration, which results in progressive muscular atrophy and weakness [1]. The most common form of SMA, 5qSMA, is 
due to the lack of survival motor neuron (SMN) protein, which is encoded by SMN1 gene located at $5 q 11.2-q 13.3$. The incidence is $1 / 6,000-1 / 10,000$ live births, and the mutation carrier rate in the general population of China is $1 / 42[2,3]$. According to the age of onset and the severity of muscle weakness, SMA is divided into four subtypes: SMA I, II, III, IV. Types I-III occur in children [1]. Due to abnormal neuromuscular functioning and impaired muscle mass, restricted motor development and decreased motor performance will persist through childhood into adulthood, resulting in various nutritional and metabolic issues $[4,5]$.

Nutritional balance and normal metabolism are the basis of maintaining health [6]. The pathological characteristics of SMA determine the increased risk of malnutrition in patients. The assessment of the nutritional status of children may also be ignored as body weight sometimes seems to be normal [7]. Furthermore, lipid metabolism is closely related to nutritional status, and lipid metabolic disorders and lack of exercise will easily lead to visceral fat accumulation and to obesity in patients with motor neuron diseases, such as SMA [8], indirectly speeding up disease progression. Nutritional status can easily be assessed through clinical measurements. However, there is no obvious clinical manifestation of abnormal lipid metabolism in the early stage of SMA, and the caregivers pay less attention to the metabolic disorders, so it is difficult to detect and intervene early. Notably, previous studies have suggested that there is obvious dyslipidemia in obese individuals $[4,9,10]$, suggesting that there may be correlation between body composition and the serum lipids, which is worth exploring.

To date, a lack of relevant research on nutritional status and lipid metabolism of SMA patients remains and hence few related health management guidelines exist. Existing studies mainly include small sample size case reports, with the subjects almost all of western nations and preliminary SMA mouse model studies $[4,9,11,12]$. Thus, whether the conclusions obtained can be extrapolated widely remains to be confirmed. In addition, as the clinical or basic research is insufficient, and these issues have received relatively low attention, research on this field for SMA needs to be expanded urgently.

To address the research gap and to gain a deep understanding of the nutritional and metabolic characteristics of SMA patients, this study aims to investigate the nutritional status, serum lipid profile, and the body composition characteristics in children with SMA, based on a hospital-based case-control study in China.

\section{Methods}

\section{Subjects and study design}

This study was a retrospective review of prospectively collected data from 91 children with SMA I-III admitted to our clinic from July, 2019 to April, 2021. All the patients were diagnosed as 5q SMA by genetic testing. Those with unclear classification and without complete clinical data were excluded. None of the children was using Nusinursen drug treatment (the only available drug for SMA in China at that time) or other drug therapies at the time of recording. All the patients had not regularly supplemented vitamin D or calcium before. The children with SMA enrolled in our study all had taken the same 
examinations on anthropometrics and clinical parameters, and some of them who had achieved three years old and been willing to cooperate also completed a comprehensive analysis for body composition by Dual-Energy X-ray Absorptiometry (DXA). In addition, to fulfill the aims of the study, we selected a group of age-, gender-matched healthy children who came to our hospital during the same period as a set of controls to compare the nutritional and dyslipidemia status of children with SMA more objectively and accurately. All subjects gave their informed consent for inclusion. The study was conducted in accordance with the Declaration of Helsinki, and the protocol was approved by the Ethics Committee of the Children's Hospital of Zhejiang University School of Medicine (Approval numbers: 2019-IRB-171).

\section{Body Measurements}

A personal file was established for each patient, including demographics, clinical characteristics, anthropometry, etc. All of the children's medical data were measured and collected by professional clinicians and clinically trained assistants. Nutritional assessments including body weight, recumbent length, and body mass index (BMI) were recorded during the clinic visit as part of the standard evaluation. Body weight was acquired using standard procedures by two trained researchers with the unified medical weight measuring instrument. Owing to the inability of standing alone or owing to scoliosis of some patients, height was uniformly measured in a supine position. Weight and height were each measured three times, and the average was used for data analysis. The anthropometric data of all the participants were described by $Z$ scores for weight for age (WAZ), height for age (HAZ), weight for height (WHZ), and BMI (BAZ/BMIZ) obtained using the World Health Organization AnthroPlus software and appropriate reference standards [13-15]. The nutritional status was divided into two parts (malnutrition and normal). Malnutrition included over-nutrition and under-nutrition, the former containing three different subtypes as wasting, underweight and stunting, which could exist at the same time in one person. Over-nutrition included two different subtypes (overweight and obesity). The judgment criteria of different nutritional status was listed as below, mainly based on the HAZ, WAZ, WHZ ( $<5$ years old), and the BMIZ ( $\geq 5$ years old), according to the Recognition and Management of Malnutrition published by the World Health Organization [13]. Under-nutrition: Wasting: WHZ<-2 (children younger than 5 years old) or BMIZ <-2 (children older than 5 years old). Stunting: HAZ<-2. Underweight: WAZ <-2. Over-nutrition: Overweight: WHZ $\ 2$ to 3 (children younger than 5 years old) or BMIZ>1 to 2 (children older than 5 years old). Obesity: WHZ囚3 (children younger than 5 years old) or BMIZ®2 (children older than 5 years old).

\section{Serum lipid levels}

Blood samples were drawn under 6-8 hours under fasting conditions. Levels of triglycerides (TG), total cholesterol (TC), Apolipoprotein A1(Apo A1), Apolipoprotein B (Apo B), Lipoprotein a (Lp (a)), high density $(\mathrm{HDL})$, and low-density (LDL) lipoprotein cholesterol were performed by an automatic biochemical analyzer (Beckman Coulter Inc., USA). Abnormal concentration of lipids or lipoproteins in the serum is collectively referred as dyslipidemia. The cut-off values of different types of dyslipidemia are provided below [16-19]: 
TG elevation is defined as TG>1.70mmol/L. TC elevation is defined as TC $>5.20 \mathrm{mmol} / \mathrm{L}$. Lipoprotein(a) elevation is defined as $\mathrm{Lp}(\mathrm{a})>500 \mathrm{mg} / \mathrm{L}$. HDL decreasion is defined as $\mathrm{HDL}<1.04 \mathrm{mmol} / \mathrm{L}$. Apo A1 decreasion is defined as Apo $A 1<1.20 \mathrm{~g} / \mathrm{L}$. LDL elevation is defined as LDL $>3.38 \mathrm{mmol} / \mathrm{L}$. Apo B elevation is defined as Apo $B>0.90 \mathrm{~g} / \mathrm{L}$.

\section{Body composition assessment}

Body composition was assessed by Dual-Energy X-ray Absorptiometry (DXA), which can quickly evaluate the body composition index of SMA patients using body mass index (BMI), lean body mass (LBM), lean body mass index (LBMI), fat mass (FM), fat mass percentage (FM\%), fat mass index (FMI) and so on. Given that children too young are not possible to quietly cooperate with the inspection, patients older than three years and able to cooperate were eligible to take this examination in our hospital. Thus, a total of 42 children with SMA II or III have finished this term. The bone health parameters (BMD and BMD-z) were also evaluated using DXA. The low bone mineral density is defined when the BMD-z of lumbar spine is less than -2 [20], which could be considered as a condition of Osteoporosis while Osteopenia, indicating low bone mass, was considered when BMD-z score lies between -1 and -2 [21]. All the indicators of body composition were compared based on the reference value provided by previous studies on healthy children in China.

\section{Statistical Analysis}

Normally distributed data are presented as the mean \pm SD and data with skewed distributions are presented with median and interquartile range (IQR). Categorical variables are presented as percentile value (\%). We conducted three types of statistical analyses here. First, the differences between two skewly distributed paired samples (e.g., serum lipid levels of the SMA disease group and the healthy control group), were evaluated using the Wilcoxon rank sum test. Differences between two sets of skewly distributed independent samples, such as anthropometric data and serum lipid levels between different gender groups in patients, were evaluated by Mann-Whitney $U$ test. The differences in rates of nutritional status and dyslipidemia between the SMA disease group and the healthy control group were compared using the Cross-tabulation Chi-square test. A Spearman correlation test was used for testing correlation between specific serum lipid indexes and body composition among SMA patients. Statistical analyses were performed using SPSS Statistics (version 26.0, BMI SPSS Inc., U.S.A.). P-values below 0.05 were considered statistically significant. Graphpad Prism 9 was used for statistical mapping.

\section{Results}

\section{Patient and control characteristics}

Table 1 includes the descriptive statistics of all patients and healthy controls. A total of 91 SMA patients were included in this analysis, with 16 (17.6\%) of SMA type I, 44 (48.4\%) of SMA type II and 31 (34.1\%) of SMA type III, respectively. Among all the SMA children, $44 \%$ were girls. More than half of the cases $(51.6 \%)$ had the ability to sit alone, although they could not walk alone. 26 cases (28.6\% of all patients) 
who were able to walk all came from SMA type III. 16 cases (17.6\% of all patients) could not sit alone and belonged to SMA type I. In disease population, children with type I were the youngest. The eldest group of children were in type III, with an average disease duration of $3.00(0.75,6.08)$ years. The median (IQR) weight of all SMA patients was $15.00(11.00,27.00) \mathrm{kg}$, the body length was $105.00(87.10,125.00) \mathrm{cm}$, and the BMI was $15.21(13.61,17.30) \mathrm{kg} / \mathrm{cm}^{2}$ (Additional file 1: Table 1), with the highest BMI level in type III and with the lowest in type I. In 91 healthy controls, on average, we observed a higher median level of body weight $(19.00 \mathrm{~kg})$, body length $(113.00 \mathrm{~cm})$ and $\mathrm{BMI}\left(16.00 \mathrm{~kg} / \mathrm{cm}^{2}\right)$.

\section{Anthropometric results and nutritional status}

In children with SMA younger than five years old $(n=46)$, the level of WHZ was $-0.57(-1.46,1.13)$, whereas in children older than five years $(n=45)$, the BMIZ level was $-1.03(-2.83,0.62)$. The overall patients' $(n=91)$ level of HAZ was $-0.61(-1.25,0.49)$ and that of WAZ $(n=91)$ was $-0.41(-1.76,0.78)$ (Additional file 1: Table 2). Based on the $Z$ scores for multiple anthropometric measurements, we observed a significant higher prevalence of malnutrition (including under- and over-nutrition) among patients (49.45\%) compared to the healthy controls (23.08\%) ( $p<0.05$, Table 2$)$. In particular, the prevalence of under-nutrition and overnutrition were both significantly higher, with a larger difference mainly observed for under-nutrition. Among different disease subtypes, patients of type III had the highest proportion of malnutrition (especially obesity), reaching $54.84 \%$, while type I patients had the highest level of under-nutrition (43.75\%, with $37.50 \%$ of underweight).

\section{Serum lipid status}

According to the results (Table 3), no significant difference was found in the prevalence of abnormally elevated TG, TC, Lp(a), LDL and Apo B between SMA group and the controls. The median levels of TC, $L p(a), L D L$ and $A p o B$ between the two groups are roughly similar. Besides, there is no obvious difference in serum lipid levels in different disease types or genders (Additional file 1: Table 3-4). However, although the median levels of TG in the SMA $(0.94(0.72,1.22) \mathrm{mmol} / \mathrm{L})$ and the control groups $(0.69(0.57,0.96)$ $\mathrm{mmol} / \mathrm{L}$ ) were both within the normal range compared with the reference value [16], a significantly statistical difference was found between the two groups ( $p<0.05$ ) (Additional file 1: Table 5). Furthermore, the levels of HDL and Apo A1 in the SMA group were significantly lower than those in the control group $(p<0.05)$. And the proportion of patients with HDL and Apo A1 lower than normal was higher than that of the controls $(p<0.01)$. Overall, a total of $56.04 \%$ of SMA children had one or more abnormal serum lipid levels, which was significantly higher than the proportion of $34.07 \%$ in the control group $(p<0.01)$.

\section{Body composition characteristics of SMA II-III}

Table 4 listed the statistics of specific indicators of body composition of 18 SMA-II patients (11 males) and 24 SMA-III patients (16 males), with the median age of $6.00(5.00,7.00)$ years old and 7.50 $(6.00,12.50)$ years old, respectively. The overall level of the fat mass percentage (FM\%) of the children was $43.38 \%(36.99 \%, 48.20 \%)$, the fat mass index (FMI) was $7.30(5.07,8.72)$, the lean body mass index (LBMI) was $7.70(6.96,9.02)$ and the bone mineral density-z score (BMD-z) was $-1.40(-2.80,-0.60)$. As 
compared with the previous reference value [21-24], the study patients' levels of the FM\% and FMI were much higher, and the levels of the LBMI and the BMD-z were lower than the reference. All body composition indicators listed in Table 4 for children with SMA type III were higher than those for type II, and significant differences between the two types were seen in FM, LBM, LBMI and BMD-z. Accordingly, 17 children had low bone mineral density, including 14(33.3\%) patients with osteoporosis and 3(7.1\%) with osteopenia. In addition, 13 of them belonged to SMA type II, accounting for $72.2 \%$ of the total type II children, which was significantly higher than the proportion of type III children with low bone mineral density $(16.7 \%)$.

\section{Correlation between Serum Lipid and Body Composition}

As shown in Fig. 1a and b, among the patients with SMA II \& III, FM\% was negatively correlated with the HDL and Apo A1 levels (Spearman $r=-0.229$ ), with a stronger correlation observed in patients with type III $(r=-0.410, p=0.047)$. Secondly, the LBMI was positively correlated with the bone mineral density $\mathrm{z}$ (BMD-z) score $(r=0.618, p<0.01)$. Third, the FMI was moderately positively correlated with the BMD-z score $(r=0.438, p=0.004)$. Furthermore, we explored the correlation between BMI and the BMD-z score and found that the two indicators were also correlated with each other ( $r=0.511, p=0.03$; Fig. 2$)$.

\section{Discussion}

In this hospital-based case-control study, we comprehensively collected the anthropometric, serum lipid and objective body composition data of children with SMA and healthy controls, which, to the best of our knowledge, is one of the few studies in this area. Overall, the prevalence of malnutrition of children with SMA was found to be significantly higher than controls, especially for under-nutrition. Moreover, SMA children are more prone to dyslipidemia. In addition, based on body composition profile, children with SMA type II \& III in China had an obviously abnormal body composition structure and low bone mineral density, supplementary correlation studies suggested that HDL/Apo A1 is negatively correlated with fat mass, while bone mineral density is positively correlated with the lean body mass.

Compared with the general Chinese children, children with SMA are sensitive to malnutrition, especially to under-nutrition [25-27] and the prevalence of under-nutrition in our patients is higher compared to patients of other nations [28, 29]. Recently, Zhou et al. [30] pointed that over $40 \%$ of children with type II \& III were at risk of malnutrition based on a case series study of 86 patients. This is roughly similar to our conclusions, but we also found the risk of obesity in children with type III may be higher than that of under-nutrition, whereas these findings are opposite in the SMA type II. This may depend on several factors. Previous research suggested that unhealthy diet, such as a high-fat, low-protein diet, may potentially lead to the high obesity prevalence of children with SMA type III who have the lightest clinical manifestations than types I and II [31]. Lack of enough physical activity as physiological requires, a large number of substances are converted into fats and accumulate in the body due to protein synthesis obstacles [1, 31, 32]. In addition, children with type I have the most severe disease phenotype, and the illness progress rapidly, which may affect function of swallowing, feeding and breathing at the fastest 
stage of growth [29, 33, 34]. Hence, they are prone to acute under-nutrition [29, 33]. In children with type II, the spine is easily affected due to the inability to walk and to the long-time sitting. Scoliosis compresses the chest and abdomen, causing digestive system problems [29, 35], then affecting appetite and eating; therefore, it is prone to insufficient nutrition intake and results more in under-nutrition.

Previously, studies have suggested that SMA patients may have dyslipidemia, and even fatty liver $[4,9$, 11,36]. We extended the study to Chinese children with SMA and found over half of them had abnormalities in serum lipids, which was partially in agreement with earlier studies. Deguise et al. [9] studied the data of children with SMA types I-III and concluded that about $37.5 \%$ of patients had at least one abnormal serum lipid index, and had liver steatosis. Under the condition that the age level of the subjects and the disease types are the same, only 2 children in our study had obvious liver steatosis, suggesting that the lipid metabolism of SMA may be affected by regions, cultural habits or populations. So far, our patients did not have obvious hyperlipidemia; however, HDL and Apo A1, negatively related to risks of cardiovascular diseases, have decreased significantly, indicating the potential of increased risk of future cardiovascular diseases. Nevertheless, whether there is no obvious TG and TC disorders due to the insufficient duration of the disease or there are still other objective factors influencing the indicators remains to be clarified. Although the mechanism of lipid metabolism disorders of SMA is unclear, studies inferred that the deficiency of SMN protein may work [4, 9]. As the dyslipidemia of the patients may be in the primary stage, with the disease progressing, the deficiency of SMN protein and the imbalanced diet and exercise, they may have more significant abnormal levels of lipids and a higher prevalence of fatty liver. Therefore, our study provided a precious opportunity to discover lipid metabolic status of SMA in China and highlighted early attention on preventing serious dyslipidemia from causing complications.

Comparison on body composition between SMA and general Chinese children showed that SMA patients truly have differences on body composition profiles with the normal [21-24]. Furthermore, $90.5 \%$ of the patients with higher FM\% indicated that they were prone to the accumulation of body fat, often leading to obesity and overweight. Moreover, studies pointed out that BMD of SMA was lower than patients with other neuromuscular disorders [20,37]. Our study found that the prevalence of Osteoporosis $(33.3 \%)$ in SMA was higher than the average level of $12.9 \%$ in the general population, alarming that SMA patients suffer from a high risk of Osteoporosis and fractures [37], which was determined by the pathological characteristics. As type II children have lost the ability of walking, and sitting for a long time, they are prone to disuse muscular atrophy and Osteoporosis due to lack of exercise [21]. The digestive system problems and insufficient nutrient intake could also potentially increase the prevalence of Osteoporosis $[12,21,37]$.

Through the additional correlation analysis, children with high FM had low level of HDL and Apo A1, while those with high lean body mass tended to have a higher BMD-z. Controversially, some studies reported that fat mass and body weight were like lean body mass and to some extent, may have a positive correlation with BMD [38-41], which we have also found in our study. However, the pathophysiology has not yet been clarified. It makes us think that appropriately gaining weight may help maintain body bone health. Therefore, in the process of nutritional intervention in children with SMA, caregivers should not 
extremely reduce their nutrient intake for fat limitation. For future research, we will continue to expand the sample size to further explore the effect of the subtypes, disease course of SMA on nutritional status, serum lipid level and the body composition. For the 91 patients, we will regularly follow up their dynamic changes of the three aspects. Patients who have received treatment will be involved to compare the differences between patients with natural disease history and after treatment.

Recently, the new SMA diagnosis and treatment management consensus emphasized the importance of multidisciplinary management, of which nutrition, including metabolism, is a core component [42]. Based on current conclusions, body composition indicates nutritional status, which interferes with metabolic processes, suggesting that adjustment of body composition can help to maintain metabolic and nutritional status of SMA. Daily diet and exercise interventions are required [43-45] to improve lean body mass, skeletal muscle mass and bone mineral density, and to reduce excess body fat mass and fat accumulation. For all patients, it is urgent to limit the intake of fat and sugar and increase the intake of coarse grains, dietary fiber, and high-quality protein to promote muscle gain [43]. Patients with overnutrition should properly control their diet, and those with under-nutrition should ensure their daily energy intake and supplement digestive enzymes and the nutrient solutions when necessary [43]. In order to prevent bone loss and Osteoporosis, milk together with calcium and vitamin $D$ are recommended to be consumed daily [46], especially for SMA type II. Moreover, appropriate zinc supplementation can help increase appetite and promote eating for patients with under-nutrition [47]. What's more, the rehabilitation and suitable exercises counts for a lot. The body's synthesis of vitamin $D$ can be promoted by outdoor activities and sun exposure [46]. And chronic exercise improved fitness [48]. It is advised to maintain moderate exercise for half an hour to one hour a day, but should circumvent fatigue [48]. For type III patients with a lighter phenotype, sports items could be enriched, for instance, conducting combined aerobic and strengthening exercises $[44,48]$.

The study is the first evaluation of the nutritional, lipid metabolism and body composition of children with SMA in China with a relatively large sample, which helps to expand the existing study scope to the possibly largest SMA population in the world. We have carried out comprehensive analysis of the three aspects of data through health controls, and explored the correlation between important nutritional metabolism indicators as much as possible, emphasizing the use of convenient and accessible nutritional indicators to monitor and help regulate lipid levels. Furthermore, there were several limitations to mention. First, our subjects are almost children under 12 years old. Thus, we may not fully grasp the situation of juvenile patients who are in puberty. As a reason that the overall sample size is limited, the number of cases of each subtype is also affected. And the current disease population was limited to the economically developed eastern regions, so the findings may not be perfectly generalized to populations in other regions. Finally, as our study is a traditional case-control study, the conclusions of causality need the long-term longitudinal follow-up.

\section{Conclusion}


To conclude, children with SMA in China have obvious nutritional imbalances, abnormal levels of serum lipid and unbalanced body composition, with high fat body mass and low bone mineral density. Further research is needed to explore the relationship between nutritional status, metabolism and body composition indicators and the possible disorders of biological pathways to direct the management and treatment of this disease. By now, we emphasize that by multidomain lifestyle management (e.g., adjusting the diet structure, supplementing daily nutrients timely, and appropriately increasing the amount of exercise) to improve the nutrition status and to adjust body composition to improve serum lipid levels as well as to keep fitness as much as possible.

\section{Abbreviations}

SMA: Spinal Muscular Atrophy; SMN: survival motor neuron; TG: triglycerides; TC: total cholesterol; Apo A1: Apolipoprotein A1; Apo B: Apolipoprotein B; BMI: body mass index; BMD: bone mineral density; BMDZ: bone mineral density-z score; BAZ/BMIZ: Z score for BMI; DXA: Dual-Energy X-ray Absorptiometry; FM\%: fat mass percentage; FMI: fat mass index; HDL: high density lipoprotein cholesterol; HAZ: Z score for height for age; LBMI: lean body mass index; Lp(a): Lipoprotein a; LDL: low-density lipoprotein cholesterol; WAZ: Z score for weight for age; WHZ: Z score for weight for height.

\section{Declarations}

\section{Acknowledgements}

The authors want to thank all the volunteers and patients participating in this study. The authors also thank Mingjuan Jin for the contribution on the statistical methods, and thank Bob and Chuiliang Li for the polish on the language in our paper.

\section{Authors' contributions}

QL, CZY and SSM conceived and designed the study. ZCY and JJH directed and checked the methodology. YJF performed the analyses and wrote the manuscript. MY, YX, JW, YH, FC, JNJ, XC, YQC and DMZ helped collect and analyze the data. SSM was responsible for organization and survey. All authors participated in the manuscript revision, and approved the final draft.

\section{Funding}

This study was supported by the Zhejiang Province Public Welfare Technology Application Research Project (LGC21H090001), the National Natural Science Foundation of China (grant number: 81801490\&81741076) and the Key Technologies Research and Development Program of Zhejiang Province (grant number: 2021C03099).

\section{Availability of data and materials}


The original contributions presented in the study are included in the article and the Additional file, further inquiries can be directed to the corresponding authors.

\section{Ethics approval and consent to participate}

This study was approved by Ethical Committee of Children's Hospital, Zhejiang University School of Medicine (2019-IRB-171). Informed consent was obtained from all participants before the study were completed.

\section{Consent for publication}

The consent for publication has been obtained from all authors.

\section{Competing interests}

No benefits in any form have been received or will be received from a commercial party related directly or indirectly to the subject of this article.

\section{Author details}

${ }^{1}$ Department of Neurology, Children's Hospital, Zhejiang University School of Medicine, National Clinical Research Center for Child Health, Hangzhou, China. ${ }^{2}$ Department of Nutrition, Children's Hospital, Zhejiang University School of Medicine, National Clinical Research Center for Child Health, Hangzhou, China. ${ }^{3}$ Children's Hospital, Zhejiang University School of Medicine, National Clinical Research Center for Child Health, Hangzhou, China. ${ }^{4}$ School of Public Health, Zhejiang University School of Medicine, Hangzhou, China. ${ }^{5}$ The Children's hospital, School of Public Health, Zhejiang University School of Medicine, National Clinical Research Center for Child Health, Hangzhou, China.

\section{References}

1. Groen EJN, Talbot K, Gillingwater TH. Advances in therapy for spinal muscular atrophy: promises and challenges. Nat Rev Neurol. 2018; 14 (4): 214-24. doi: 10.1038/nrneurol.2018.4

2. Verhaart IEC, Robertson A, Wilson IJ, Aartsma-Rus A, Cameron S, Jones CC, et al. Prevalence, incidence and carrier frequency of $5 q$-linked spinal muscular atrophy - a literature review. Orphanet $J$ Rare Dis. 2017; 12 (1): 124. doi: 10.1186/s13023-017-0671-8

3. Su YN, Hung CC, Lin SY, Chen FY, Chern JP, Tsai C, et al. Carrier screening for spinal muscular atrophy (SMA) in 107,611 pregnant women during the period 2005-2009: a prospective population-based cohort study. PLoS One. 2011; 6 (2): e17067. doi: 10.1371/journal.pone.0017067

4. Li YJ, Chen TH, Wu YZ, Tseng YH. Metabolic and Nutritional Issues Associated with Spinal Muscular Atrophy. Nutrients. 2020; 12 (12): 3842. doi: 10.3390/nu12123842

5. Norman K, Pichard C, Lochs H, Pirlich M. Prognostic impact of disease-related malnutrition. Clin Nutr. 2008; 27 (1): 5-15. doi: 10.1016/j.clnu.2007.10.007 
6. Blum J, Epstein R, Watts S, Thalacker-Mercer A. Importance of Nutrient Availability and Metabolism for Skeletal Muscle Regeneration. Front Physiol. 2021; 12: 696018. doi: 10.3389/fphys.2021.696018

7. Mehta NM, Raphael B, Guteirrez IM, Quinn N, Mitchell PD, Litman HJ, et al. Comparison of body composition assessment methods in pediatric intestinal failure. J Pediatr Gastroenterol Nutr. 2014; 59 (1): 99-105. doi: 10.1097/MPG.0000000000000364

8. Schmitt F, Hussain G, Dupuis L, Loeffler JP, Henriques A. A plural role for lipids in motor neuron diseases: energy, signaling and structure. Front Cell Neurosci. 2014; 8: 25. doi: 10.3389/fncel.2014.00025

9. Deguise MO, Baranello G, Mastella C, Beauvais A, Michaud J, Leone A, et al. Abnormal fatty acid metabolism is a core component of spinal muscular atrophy. Ann Clin Transl Neurol. 2019; 6 (8): 1519-32. doi: 10.1002/acn3.50855

10. Watson KS, Boukhloufi I, Bowerman M, Parson SH. The Relationship between Body Composition, Fatty Acid Metabolism and Diet in Spinal Muscular Atrophy. Brain Sci. 2021; 11 (2): 131. doi: 10.3390/brainsci11020131

11. Zolkipli Z, Sherlock M, Biggar WD, Taylor G, Hutchison JS, Peliowski A, et al. Abnormal fatty acid metabolism in spinal muscular atrophy may predispose to perioperative risks. Eur J Paediatr Neurol. 2012; 16 (5): 549-53. doi: 10.1016/j.ejpn.2012.01.004

12. Mehta NM, Newman H, Tarrant S, Graham RJ. Nutritional Status and Nutrient Intake Challenges in Children With Spinal Muscular Atrophy. Pediatr Neurol. 2016; 57: 80-3. doi:

10.1016/j.pediatrneurol.2015.12.015

13. WHO Multicentre Growth Reference Study Group. WHO Child Growth Standards: Length/height- forage, weight-for-age, weight-for-length, weight-for-height, and body mass index-for-age: Methods and development. Geneva: World Health Organization.2006;312.

14. de Onis M, Onyango AW, Borghi E, Siyam A, Nishida C, Siekmann J. Development of a WHO growth reference for school-aged children and adolescents. Bull World Health Organ. 2007; 85 (9): 660-7. doi: $10.2471 /$ blt.07.043497

15. Cole TJ, Bellizzi MC, Flegal KM, Dietz WH. Establishing a standard definition for child overweight and obesity worldwide: international survey. BMJ. 2000; 320 (7244): 1240-3. doi:

10.1136/bmj.320.7244.1240

16. Jellinger PS, Handelsman Y, Rosenblit PD, Bloomgarden ZT, Fonseca VA, Garber AJ, et al. American Association of Clinical Endocrinologists and American College of Emdocrinology Guidelines for Management of Dyslipidemia and Prevention of Cardiovascular Disease. Endocr Pract. 2017; 23 (Suppl 2): 1-87. doi: 10.4158/EP171764.APPGL

17. Saeed A, Kinoush S, Virani SS. Lipoprotein (a): Recent Updates on a Unique Lipoprotein. Curr Atheroscler Rep. 2021; 23 (8): 41. doi: 10.1007/s11883-021-00940-5

18. Li S, Liu X, Okada T, Iwata F, Hara M, Harada K. Serum lipid profile in obese children in China. Pediatr Int. 2004; 46 (4): 425-8. doi: 10.1111/j.1442-200x.2004.01908.x 
19. Mach F, Baigent C, Catapano AL, Koskinas KC, Casula M, Badimon L, et al. 2019 ESC/EAS Guidelines for the management of dyslipidaemias: lipid modification to reduce cardiovascular risk. Eur Heart J. 2020; 41 (1): 111-88. doi: 10.1093/eurheartj/ehz455

20. Khatri IA, Chaudhry US, Seikaly MG, Browne RH, lannaccone ST. Low bone mineral density in spinal muscular atrophy. J Clin Neuromuscul Dis. 2008; 10 (1): 11-7. doi:

10.1097/CND.0b013e318183e0fa

21. Saggese G, Baroncelli GI, Bertelloni S. Osteoporosis in children and adolescents: diagnosis, risk factors, and prevention. J Pediatr Endocrinol Metab. 2001; 17 (7): 833-59. doi: 10.1515/jpem.2001.14.7.833

22. Liu J, Yan Y, Xi B, Huang G, Mi J, China Child and Adolescent Cardiovascular Health (CCACH) Study Group. Skeletal muscle reference for Chinese children and adolescents. J Cachexia Sarcopenia Muscle. 2019; 10 (1): 155-64. doi: 10.1002/jcsm. 12361

23. Iwata F, Hara M, Okada T, Harada K, Li S. Body fat ratios in urban Chinese children. Pediatr Int. 2003; 45 (2): 190-2. doi: 10.1046/j.1442-200x.2003.01688.x

24. Guo B, Xu Y, Gong J, Tang Y, Shang J, Xu H. Reference data and percentile curves of body composition measured with dual energy $\mathrm{X}$-ray absorptiometry in healthy Chinese children and adolescents. J Bone Miner Metab. 2015; 33 (5): 530-9. doi: 10.1007/s00774-014-0615-5

25. Zong XN, Li H, Zhang YQ, Wu HH. Child nutrition to new stage in China: evidence from a series of national surveys, 1985-2015. BMC Public Health. 2019; 19 (1): 402. doi: 10.1186/s12889-019-6699-z

26. Yang B, Huang X, Liu Q, Tang S, Story M, Chen Y, et al. Child Nutrition Trends Over the Past Two Decades and Challenges for Achieving Nutrition SDGs and National Targets in China. Int J Environ Res Public Health. 2020; 17 (4): 1129. doi: 10.3390/ijerph17041129

27. Zhang YQ, Li H, Wu HH, Zong XN. Stunting, wasting, overweight and their coexistence among children under 7 years in the context of the social rapidly developing: Findings from a populationbased survey in nine cities of China in 2016. PLoS One. 2021; 16 (1): e0245455. doi: 10.1371/journal.pone.0245455

28. Martinez EE, Quinn N, Arouchon K, Anzaldi R, Tarrant S, Ma NS, et al. Comprehensive nutritional and metabolic assessment in patients with spinal muscular atrophy: Opportunity for an individualized approach. Neuromuscul Disord. 2018; 28 (6): 512-9. doi: 10.1016/j.nmd.2018.03.009

29. Moore GE, Lindenmayer AW, McConchie GA, Ryan MM, Davidson ZE. Describing nutrition in spinal muscular atrophy: A systematic review. Neuromuscul Disord. 2016; 26 (7): 395-404. doi: 10.1016/j.nmd.2016.05.005

30. Zhou Y, Chen J, Gong X, Lu Z, Hua H, Zhu X, et al. Nutrition status survey of type 2 and 3 spinal muscular atrophy in Chinese population. Nutr Neurosci. 2021; 23: 1-7. doi:

10.1080/1028415X.2020.1871212

31. Sproule DM, Montes J, Dunaway S, Montgomery M, Battista V, Koenigsberger D, et al. Adiposity is increased among high-functioning, non-ambulatory patients with spinal muscular atrophy. Neuromuscul Disord. 2010; 20 (7): 448-52. doi: 10.1016/j.nmd.2010.05.013 
32. Maharjan BR, Martinez-Huenchullan SF, Mclennan SV, Twigg SM, Williams PF. Exercise induces favorable metabolic changes in white adipose tissue preventing high-fat diet obesity. Physiol Rep. 2021; 9 (16): e14929. doi: 10.14814/phy2.14929

33. Corsello A, Scatigno L, Pascuzzi MC, Calcaterra V, Dilillo D, Vizzuso S, et al. Nutritional, Gastrointestinal and Endo-Metabolic Challenges in the Management of Children with Spinal Muscular Atrophy Type 1. Nutrients. 2021; 13 (7): 2400. doi: 10.3390/nu13072400

34. van der Heul AMB, Cuppen I, Wadman RI, Asselman F, Schoenmakers MAGC, van de Woude DR, et al. Feeding and Swallowing Problems in Infants with Spinal Muscular Atrophy Type 1: an Observational Study. J Neuromuscul Dis. 2020; 7 (3): 323-30. doi: 10.3233/JND-190465

35. Messina S, Pane M, De Rose P, Vasta I, Sorleti D, Aloysius A, et al. Feeding problems and malnutrition in spinal muscular atrophy type II. Neuromuscul Disord. 2008; 18 (5): 389-93. doi:

10.1016/j.nmd.2008.02.008

36. Deguise MO, Chehade L, Kothary R. Metabolic Dysfunction in Spinal Muscular Atrophy. Int J Mol Sci. 2021; 22 (1): 5913. doi: 10.3390/ijms22115913

37. Wasserman HM, Hornung LN, Stenger PJ, Rutter MM, Wong BL, Rybalsky I, et al. Low bone mineral density and fractures are highly prevalent in pediatric patients with spinal muscular atrophy regardless of disease severity. Neuromuscul Disord. 2017; 27 (4): 331-7. doi: 10.1016/j.nmd.2017.01.019

38. Seo YG, Kim Y, Lim H, Kang MJ, Park KH. Relationship Between Bone Mineral Density and Body Composition According to Obesity Status in Children. Endocr Pract. 2021; 16: S1530-891X(21)011010. doi: 10.1016/j.eprac.2021.06.006

39. Tomlinson DJ, Erskine RM, Morse Cl, Onambélé GL. Body Fat Percentage, Body Mass Index, Fat Mass Index and the Ageing Bone: Their Singular and Combined Roles Linked to Physical Activity and Diet. Nutrients. 2019; 11 (1): 195. doi: 10.3390/nu11010195

40. Deng G, Yin L, Li K, Hu B, Cheng X, Wang L, et al. Relationships between anthropometric adiposity indexes and bone mineral density in a cross-sectional Chinese study. Spine J. 2021; 21 (2): 332-42. doi: 10.1016/j.spinee.2020.10.019

41. Kim YS, Han JJ, Lee J, Choi HS, Kim JH, Lee T. The correlation between bone mineral density/trabecular bone score and body mass index, height, and weight. Osteoporos Sarcopenia. 2017; 3 (2): 98-103. doi: 10.1016/j.afos.2017.02.001

42. Mercuri E, Finkel RS, Muntoni F, Wirth B, Montes J, Main M, et al. Diagnosis and management of spinal muscular atrophy: Part 1: Recommendations for diagnosis, rehabilitation, orthopedic and nutritional care. Neuromuscul Disord. 2018; 28 (2): 103-15. doi: 10.1016/j.nmd.2017.11.005

43. Willoughby D, Hewlings S, Kalman D. Body Composition Changes in Weight Loss: Strategies and Supplementation for Maintaining Lean Body Mass, a Brief Review. Nutrients. 2018; 10 (12): 1876. doi: $10.3390 /$ nu10121876

44. Bartels B, Montes J, van der Pol WL, de Groot JF. Physical exercise training for type 3 spinal muscular atrophy. Cochrane Database Syst Rev. 2019; 3 (3): CD012120. doi: 
10.1002/14651858.CD012120.pub2

45. Stoner L, Rowlands D, Morrison A, Credeur D, Hamlin M, Gaffney K, et al. Efficacy of Exercise Intervention for Weight Loss in Overweight and Obese Adolescents: Meta-Analysis and Implications. Sports Med. 2016; 46 (11): 1737-51. doi: 10.1007/s40279-016-0537-6

46. Xu H, Zhao Z, Wang H, Ding $M$, Zhou A, Wang X, et al. Bone mineral density of the spine in 11,898 Chinese infants and young children: a cross-sectional study. PLoS One. 2013; 8 (12): e82098. doi: 10.1371/journal.pone.0082098

47. Chao HC, Chang YJ, Huang WL. Cut-off Serum Zinc Concentration Affecting the Appetite, Growth, and Nutrition Status of Undernourished Children Supplemented With Zinc. Nutr Clin Pract. 2018; 33 (5): 701-10. doi: 10.1002/ncp.10079

48. Ng SY, Manta A, Ljubicic V. Exercise biology of neuromuscular disorders. Appl Physiol Nutr Metab. 2018; 43 (11): 1194-206. doi: 10.1139/apnm-2018-0229

\section{Tables}

Table 1 Basic Demographic and Clinical Characteristics of all the SMA patients and healthy controls.

Table 2 Nutritional status of SMA patients and age-, gender-matched hospital-based controls.

\begin{tabular}{|c|c|c|c|c|c|}
\hline \multirow[t]{2}{*}{ Nutritional status ${ }^{a}$} & \multicolumn{3}{|c|}{ Overall SMA $(\mathrm{N}=91)$} & \multirow[b]{2}{*}{ Control $(\mathrm{N}=91)$} & \multirow[b]{2}{*}{ P-value ${ }^{b}$} \\
\hline & SMA-I & SMA-II & SMA-III & & \\
\hline Under-nutrition (n, \%) & $7(43.75)$ & $17(38.63)$ & $6(19.35)$ & $8(8.79)$ & $<0.01$ \\
\hline Wasting & $5(31.25)$ & $11(25.00)$ & $5(16.13)$ & $4(4.40)$ & $<0.01$ \\
\hline Underweight & $6(37.50)$ & $11(25.00)$ & $4(12.90)$ & $0(0.00)$ & $<0.01$ \\
\hline Stunting & $1(6.25)$ & $8(18.18)$ & $0(0.00)$ & $4(4.40)$ & 0.25 \\
\hline Over-nutrition (n, \%) & $1(6.25)$ & $3(6.82)$ & $11(35.48)$ & 13(14.29) & 0.84 \\
\hline Overweight & $1(6.25)$ & $1(2.27)$ & $4(12.90)$ & $8(8.79)$ & 0.78 \\
\hline Obese & $0(0.00)$ & $2(4.55)$ & $7(22.58)$ & $5(5.49)$ & 0.41 \\
\hline Normal (n, \%) & $8(50.00)$ & $24(54.55)$ & $14(45.16)$ & $70(76.92)$ & $<0.01$ \\
\hline
\end{tabular}

\footnotetext{
${ }^{a}$ Nutritional status includes malnutrition and the normal. Malnutrition is divided into over-nutrition and under-nutrition. Under-nutrition includes wasting, underweight and stunting, while over-nutrition includes overweight and obese. The percentages of different disease types were calculated within the corresponding SMA type, respectively.
} 
Characteristics

Overall SMA

Control

SMA-I SMA-II SMA-III

\begin{tabular}{|c|c|c|c|c|}
\hline Total (n, \%) & 16(17.6) & \multicolumn{2}{|c|}{$31(34.0)$} & $91(100.0)$ \\
\hline \multicolumn{5}{|l|}{$\operatorname{Sex}(n, \%)$} \\
\hline Female & $6(37.5)$ & $2(50.0)$ & 12(38.7) & $40(44.0)$ \\
\hline Male & $10(62.5)$ & $22(50.0)$ & 19(61.3) & $51(56.0)$ \\
\hline Age (y), median (IQR) & $0.8(0.4,2.6)$ & $4.1(2.6,6.5)$ & $6.8(3.3,9.7)$ & $5.0(2.1,7.9)$ \\
\hline Weight (kg), median (IQR) & $\begin{array}{l}8.4(7.1,11.5) \\
35.0)\end{array}$ & $14.0(12.0,22.0)$ & 24.0(17.0, & $19.0(13.4,28.0)$ \\
\hline Length $(\mathrm{cm})$, median (IQR) & \multicolumn{3}{|c|}{$\begin{array}{l}77.0(70.1,100.8) \quad 101.0(89.3,120.4) \\
119.0(100.0,137.0)\end{array}$} & $\begin{array}{l}113.0(86.0 \\
132.0)\end{array}$ \\
\hline $\mathrm{BMI}\left(\mathrm{kg} / \mathrm{cm}^{2}\right)$, median (IQR) & $\begin{array}{l}14.4(13.3,15.0) \\
18.9)\end{array}$ & $15.2(13.6,16.6)$ & 16.5(14.1, & $16.0(14.7,17.6)$ \\
\hline $\begin{array}{l}\text { Course of disease (y), } \\
\text { median (IQR) }\end{array}$ & $0.4(0.2,1.9)$ & \multicolumn{2}{|l|}{$3.5(1.4,5.8)$} & NA \\
\hline
\end{tabular}

Ambulant ability (n, \%)

Walkers

$0(0.0)$

$0(0.0)$

26(83.9)

NA

Sitters

$0(0.0)$

42(95.5)

$5(16.1)$

NA

Non-sitters

16(100.0) 2(4.5)

$0(0.0)$

NA

${ }^{\mathrm{b}}$ The significant difference in rate between overall SMA patients (including type I-type III) and the control group is compared using the cross-tabulation method.

According to the different definitions listed in the methodology section, the subtypes of under-nutrition and over-nutrition can exist in one patient.

Table 3 Comparison among the percentages of serum lipids disfunction between SMA patients and the hospital-based control. 


\begin{tabular}{|c|c|c|c|}
\hline Serum lipids ${ }^{a}$ & Overall SMA $(\mathrm{N}=91)$ & Control $(\mathrm{N}=91)$ & P-value \\
\hline $\mathrm{TG}>1.70 \mathrm{mmol} / \mathrm{L}$ & 12(13.19) & $5(5.49)$ & 0.12 \\
\hline $\mathrm{TC}>5.20 \mathrm{mmol} / \mathrm{L}$ & 12(13.19) & $17(18.68)$ & 0.42 \\
\hline Lipoprotein(a) >500mg/L & $6(6.59)$ & $6(6.59)$ & 1.00 \\
\hline $\mathrm{HDL}<1.04 \mathrm{mmol} / \mathrm{L}$ & $24(26.37)$ & $7(7.69)$ & $<0.01$ \\
\hline LDL $>3.38 \mathrm{mmol} / \mathrm{L}$ & $11(12.09)$ & $9(9.89)$ & 0.81 \\
\hline ApoAl<1.2g/L & $38(41.76)$ & $6(6.59)$ & $<0.01$ \\
\hline$A p o B>0.9 \mathrm{~g} / \mathrm{L}$ & $19(20.88)$ & $17(18.68)$ & 0.85 \\
\hline Abnormal serum lipid items $\geq 1 \quad(n, \%)$ & $51(56.04)$ & $31(34.07)$ & $<0.01$ \\
\hline Abnormal serum lipid items $\geq 2 \quad(n, \%)$ & $45(49.45)$ & $30(32.97)$ & 0.02 \\
\hline
\end{tabular}

${ }^{a}$ Recorded in the table are all the clinically significant serum lipid abnormalities in the studied population. The judgment standard is determined based on previous studies on serum lipids and standards issued by the WHO organization.

Table 4 Body composition results of SMA-II and SMA-III patients.

\begin{tabular}{|llcc|}
\hline Variable & SMA-II (N=18) & SMA-III (N=24) & \multicolumn{1}{c|}{ P-value } \\
\hline FM (kg), median (IQR) & $8.67(5.41,13.87)$ & $13.77(7.85,16.56)$ & $\square 0.01$ \\
\hline FM\%(\%), median (IQR) & $45.19(38.53,49.45)$ & $46.08(38.53,50.94)$ & 0.36 \\
\hline FMI, median (IQR) & $6.96(4.78,8.44)$ & $7.83(5.13,9.49)$ & 0.21 \\
\hline LBM(kg), median (IQR) & $8.34(7.08,12.20)$ & $13.68(11.15,21.05)$ & $\square 0.01$ \\
\hline LBMI, median (IQR) & $7.01(6.23,7.43)$ & $8.56(7.74,9.88)$ & $\square 0.01$ \\
\hline BMD (g/cm $\left.{ }^{2}\right)$, median (IQR) & $0.75(0.41,0.85)$ & $0.79(0.66,0.89)$ & 0.16 \\
\hline BMD-z, median (IQR) & $-2.90(-3.68,-1.85)$ & $-0.95(-1.40,-0.10)$ & 00.01 \\
\hline
\end{tabular}

Body composition was examined by Dual-Energy X-ray Absorptiometry (DXA), which is regarded as the gold standard method for analyzing body composition. In view that the DXA requires a period of stillness, we only performed this examination on some of the children who were over 3 years old and able to coorperate. Thus, we displayed all 42 patients' results here, including 18 patients with SMA-II and 24 with SMA-III, with the median age of $6.00(5.00,7.00)$ years old and $7.50(6.00,12.50)$ years old, respectively, and 11 males of type II and 16 males of type III.

\section{Figures}



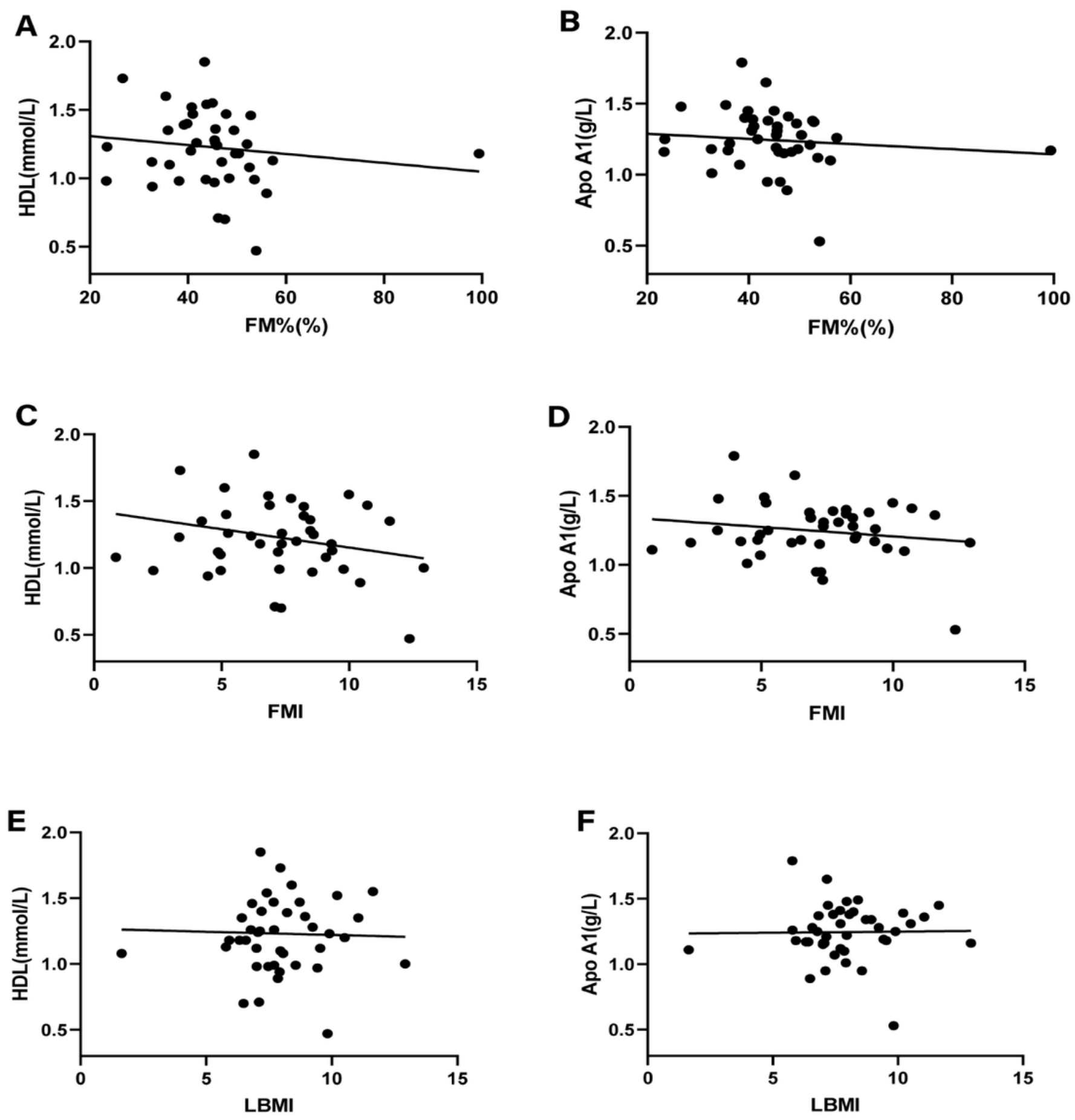

Figure 1

Bivariate correlation analysis between serum lipid levels and body composition. a Correlation analysis between FM\% and HDL. b Correlation analysis between FM\% and Apo A1. c Correlation analysis between FMI and HDL. d Correlation analysis between FMI and Apo A1. e Correlation analysis between LBMI and HDL. f Correlation analysis between LBMI and Apo A1. 


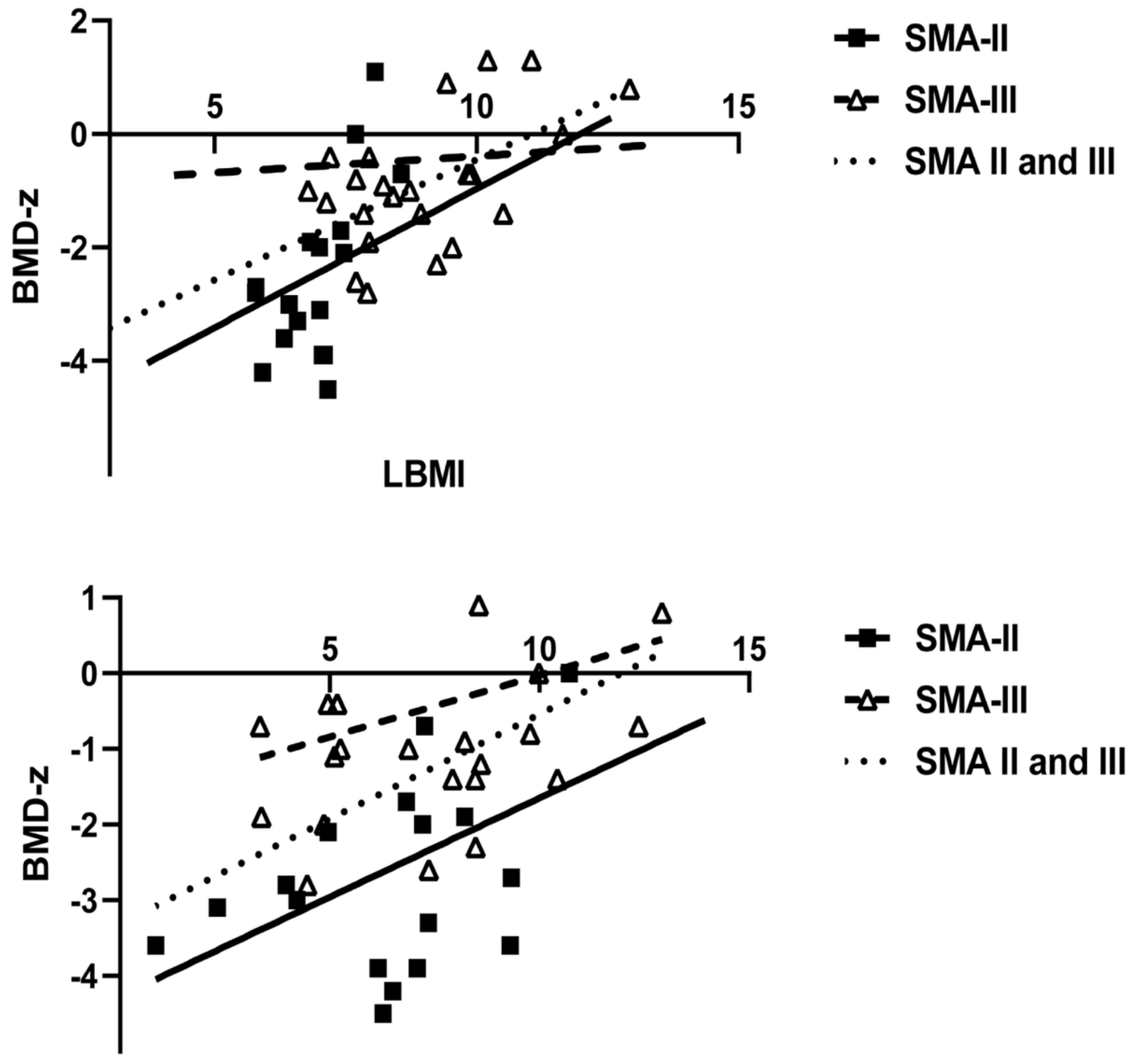

FMI

Figure 2

Bivariate correlation analysis between body composition indicators. a Bivariate correlation analysis between the body composition LBMI and the bone mineral density z-score(BMD-z). b Bivariate correlation analysis between the body composition FMl and the bone mineral density z-score(BMD-z). (Closed squares and closed line: patients with SMA type 2; the dashed line and hollow triangles: patients with SMA type 3; the dotted line: the overall patients. 


\section{Supplementary Files}

This is a list of supplementary files associated with this preprint. Click to download.

- Additionalfile1.docx 